population in Ireland. Targeted interventions, such as preventive messaging in collaboration with key LGBT + community and health service partners, may be warranted to reduce the burden of AUD among MSM.

\section{P66 TRANSLATION AND VALIDATION OF THE SWAHILI WARWICK EDINBURGH MENTAL WELLBEING SCALE (WEMWBS) AND DISTRIBUTION OF MENTAL WELLBEING IN ADOLESCENTS AND ADULTS TAKING PART IN THE GIRLS' EDUCATION CHALLENGE PROJECT IN TANZANIA}

\begin{abstract}
${ }^{1}$ Louise Banham, ${ }^{2}$ Elizabeth Bruno-McClung, ${ }^{3}$ Deus Kapinga, ${ }^{2}$ Oyinlola Oyebode*, ${ }^{2}$ Sarah Stewart-Brown, ${ }^{4}$ Manuel Torres-Sahli, ${ }^{3}$ Lydia Wilbard, ${ }^{2}$ Rebecca Willans. ${ }^{1}$ UK Foreign, Commonwealth and Development Office, Dar Es Salaam, Tanzania; ${ }^{2}$ Warwick Medical School, University of Warwick, Coventry, UK; ${ }^{3}$ CAMFED Campaign for female education, Dar Es Salaam, Tanzania; ${ }^{4}$ School of Social Sciences and Humanities, University of Loughborough, Loughborough, UK
\end{abstract}

\subsection{6/jech-2021-SSMabstracts. 154}

Background The Warwick Edinburgh Mental Wellbeing Scale (WEMWBS) is validated for measuring mental wellbeing in UK populations aged $11+$ and has been translated into $30+$ languages. The primary aim of this study was to translate and validate WEMWBS for use in Swahili-speaking populations. This will facilitate measurement and understanding of wellbeing in these populations, evaluation of policy and practice, and enable international comparisons. The secondary aim was to describe mental wellbeing in the participants of the Girls' Education Challenge (GEC) project in Tanzania, run by CAMFED and funded through the UK's Foreign, Commonwealth and Development Office. Specifically, to examine sociodemographic characteristics associated with higher and lower wellbeing in this population.

Methods We created a short questionnaire including WEMWBS and similar scales for comparison, socio-demographic information and self-reported health. This was translated into Swahili using gold standard methodology. We aimed to use this tool to collect data from secondary school students (girls and boys), learner guides, teacher mentors and teachers taking part in the GEC programme in Tanzania. Quantitative data analysis examined internal consistency of WEMWBS, correlation with comparator scales and confirmatory factor analysis. Qualitative work to assess acceptability and comprehensibility of WEMWBS and conceptual understanding of mental wellbeing was carried out through focus groups with GEC participants. These were audio-taped, transcribed and analysed thematically. Finally, we used multivariable logistic regression to explore associations between individual characteristics and 'low' and 'high' mental wellbeing, defined as the lowest and highest quartile of WEMWBS scores.

Results 3052 students were recruited into the study and 574 adults. Both WEMWBS and its short form met quantitative test of reliability and validity. They were correlated with comparator scales and met the criteria to determine that they were measuring one factor. Overall, WEMWBS seemed applicable, understood and relevant to the focus groups of students, learner guides and teachers. For students in the Girls Education Challenge supported government schools: being male, being urban, the absence of markers of marginality and better self-reported health were all significantly associated with better mental wellbeing. Mental wellbeing is higher in students in the final two 'forms' of school compared with the first two. For adults: being urban and better self-reported health were associated with better mental wellbeing.

Conclusion The Swahili translation of WEMWBS is available for use in Swahili speaking populations. Further work to explore how to intervene to increase mental wellbeing in vulnerable GEC participants is needed.

\section{\begin{tabular}{l|l} 
P67 & CLASS-BASED NEIGHBOURHOOD MINORITY STATUS
\end{tabular} PREDICTS MENTAL HEALTH, FOR CULTURAL BUT NOT ECONOMIC COMPONENTS OF CLASS}

${ }^{1}$ Christopher WN Saville*, ${ }^{2}$ Robin Mann. 'North Wales Clinical Psychology Programme, School of Psychology, Bangor University, Bangor, UK; ${ }^{2}$ School of History, Philosophy, and Social Sciences, Bangor University, Bangor, UK

\subsection{6/jech-2021-SSMabstracts. 155}

Background A considerable literature of ethnic density studies links mental health to ethnic minority status in one's neighbourhood. This design has also been applied to other types of identity, such as sexual minorities and Brexit identities, but surprisingly little attention has been paid to class-based identities. Bourdieu decomposes class into several distinct, but mutually reinforcing, forms of capital. Here, we examine whether a 'class density' association with mental health can be found for economic capital - people's material assets and cultural capital - symbolic signifiers of class such as tastes and interests.

Methods Multi-level regression with post-stratification was used to make area-level estimates of cultural and economic capital for each middle super output area in Wales using data from the National Survey for Wales 2017/18 ( $N=11381)$. Mixed effects models were fitted to economic capital, operationalised using respondents' income, house ownership, and material deprivation, and cultural capital, operationalised using items on attendance at a variety of artistic, cultural, and heritage activities. Terms for demographic and area-level variables, as well as random effects of middle super output area were estimated and post-stratified using census to create estimates of area-level economic and cultural capital for all Welsh middle super output areas. These estimates were linked to independent individual-level data from the 2018/19 edition of the survey $(\mathrm{N}=4058)$. Mixed effects models containing individuallevel capital, area-level capital, and their interaction were fitted, predicting whether respondents reported a mental health problem. Models were fitted unadjusted, adjusted for age and gender, adjusted for the other form of capital on the individual and area levels, and adjusted for all these and the other capital's cross-level interaction. Multiple imputation was used to account for missing data.

Results For cultural capital, a cross-level interaction was found where area-level cultural capital was protective in respondents reporting higher levels of individual-level cultural capital, but a risk factor for mental health problems in those with low individual-level cultural capital (odds ratio $=.83, \mathrm{CI}_{95 \%}=.74$ .93). No such relationship was found for economic capital (odds ratio $=.96, \mathrm{CI}_{95 \%}=.88-1.06$ ). These results remained robust in the adjusted models.

Conclusion The presence of a class density association with mental health for cultural capital but not economic capital 
suggests that class-based identities can also lead to density associations with mental health, but that it is the symbolic signifiers of class, and not material aspects, which activate class identities in this way.

\section{P68 ANTIDEPRESSANT AND ANTIPSYCHOTIC DRUG PRESCRIBING AND COMPLICATIONS OF DIABETES: A SYSTEMATIC REVIEW OF OBSERVATIONAL STUDIES}

Charlotte Greene*, Hanna Ward-Penny, Sarah Wild, Caroline Jackson. Usher Institute of Population Health Sciences and Informatics, The University of Edinburgh, Edinburgh, UK

\subsection{6/jech-2021-SSMabstracts. 156}

Background The prescribing of antidepressant and antipsychotic drugs is increasing worldwide for a range of indications including mental ill-health. Numerous side effects have been associated with these drugs, including increased risk of cardiovascular complications. People with diabetes are more likely to be prescribed these drugs but few studies have investigated the effect of these drugs in this specific population. We therefore conducted a systematic review of studies that investigated the association between antidepressant and/or antipsychotic drug prescribing and vascular complications or mortality in people with diabetes.

Methods We systematically searched PubMed, EMBASE, and PsycINFO for observational studies examining the association between use of antidepressant and/or antipsychotic drug use and vascular disease (including cardiovascular disease and microvascular complications) and all-cause and cause-specific mortality among adults with type 1 or 2 diabetes. Secondary outcomes of interest included cardiometabolic risk factors. Data extraction was performed independently by two reviewers using standardised data collection forms.

Results Our search retrieved 9,833 articles, of which 13 were included in the review. Studies were heterogenous in design, population, and outcomes. Six studies were cohort design, two were case control, and five were cross-sectional. Some studies included an unselected population and others included only people with particular mental illnesses or excluded people with mental illness. Six studies reported on vascular complications, six studies reported on cardiometabolic risk factors (including glycaemic control), and one study reported on both vascular complications and cardiometabolic risk factors. Study population size ranged from 265 to 241,787. Whilst study heterogeneity makes comparability difficult and findings were mixed, there was some evidence that antidepressant and antipsychotic drugs may lead to a reduced risk of cardiovascular morbidity, possibly more so among women than men. There was also evidence that antidepressant use may lead to a reduced risk of diabetic retinopathy in women. There was evidence that antidepressants can lead to improved glycaemic control among people with depression, but there may be an increased risk of hospitalisation for hyperglycaemia in people using antipsychotics.

Conclusion Few studies have examined the effect of antidepressant and antipsychotic drugs on complications of diabetes, with mixed findings reported. These drugs may have a protective effect potentially mediated by their therapeutic effects on mental health and adherence to lifestyle advice and other treatment. It is also possible that there is effect modification by sex, with some protective effects observed in women but not in men. More research on this topic is required.

\section{P69 A COMPREHENSIVE SYSTEMATIC REVIEW AND MULTILEVEL META-ANALYSIS OF THE ETHNIC DENSITY EFFECT IN PSYCHOSIS}

${ }^{1}$ Sophie J Baker*, ${ }^{1,2}$ Mike Jackson, ${ }^{3,4}$ Hannah Jongsma, ${ }^{1}$ Christopher WN Saville. 'School of Psychology, Bangor University, Bangor, UK; ${ }^{2}$ Betsi Cadwaladr University Health Board, Bangor, UK; ${ }^{3}$ Centre for Transcultural Psychiatry Veldzicht, Balkbrug, The Netherlands; ${ }^{4}$ Department of Psychiatry, University of Groningen and University Medical Centre Groningen, Groningen, The Netherlands

\subsection{6/jech-2021-SSMabstracts. 157}

Background An ethnic density effect in psychosis has been observed whereby the risk of psychosis in minority group individuals is inversely related to the neighbourhood-level proportion of others belonging to the same group. However, there is conflicting evidence over whether this effect differs between minority groups and limited investigation into other moderators. We aimed to conduct a comprehensive systematic review and meta-analysis of the ethnic density effect in psychosis and examine moderators.

Methods Four databases were systematically searched. A narrative review was conducted, and a three-level meta-analysis was performed. The potential moderating effect of crudely and specifically defined minority groups was assessed. Country, time, area size, and whether studies used clinical or non-clinical outcomes were also tested as moderators.

Results Thirty-two studies were included in the narrative review and ten in the meta-analysis. A ten percentage-point decrease in own-group density was associated with a $20 \%$ increase in psychosis risk $\left[\mathrm{OR}=1.20 \quad\left(\mathrm{CI}_{95 \%}=1.09-1.32\right)\right.$, $p<0.001]$. The pooled effect was moderated by crudely defined minority groups $\left[F_{6,68}=6.86, p<0.001\right]$, with the strongest associations observed in Black populations, followed by a White Other sample. Greater heterogeneity was observed when specific minority groups were assessed $\left[F_{25,49}=7.26\right.$, $p<0.001]$.

Conclusion This is the first review to provide meta-analytic evidence that the risk of psychosis posed by lower own-group density areas is not equally distributed across minority groups. The most robust associations were observed in Black individuals. Heterogeneity in effect sizes may reflect distinctive social experiences of specific minority groups. Mechanisms are discussed, along with the implications of findings and suggestions for future research.

\section{P70 SUBSTANCE USE AMONG SEXUAL MINORITIES IN THE USA - LINKS TO SOCIOECONOMIC VULNERABILITY AND UNMET NEED FOR MENTAL HEALTH TREATMENT}

${ }^{1}$ Jessica Neicun*, ${ }^{2}$ Bastian Rosner. 'International Health, Maastricht University, Maastricht, The Netherlands; ${ }^{2}$ Institute of Public Health, Cambridge University, Cambridge, UK

\subsection{6/jech-2021-SSMabstracts. 158}

Background Sexual minority groups (SM) have specific substance use patterns that remain widely unknown. We investigated the potential association between substance use among SM adults in the United States (USA) and social inequality, with an additional focus on disparities in unmet need for mental health treatment. 